\section{ARTIFICIAL CELLS}

\section{Converting light into ATP}

Angew. Chem. https://doi.org/10.1002/

ange.201813963 (2019)

Artificial cells are compartmentalized structures that can mimic essential functions of their biological counterpart. They have been useful to understand and recreate minimalistic metabolic pathways and the structure of protocells. Now, Chen and co-workers report artificial cells that can convert light into biochemical energy in the system is composed of two artificial cells, one that converts light into a proton gradient and one that converts this gradient into ATP.

To convert light into a proton gradient, the researchers self-assemble an artificial cell made of packed Au-Ag nanorods with an embedded bacteriorhodopsin. The nanorods induce a preferential orientation of the bacteriorhodopsin. As a result, on irradiation, the bacteriorhodopsin pumps protons from inside the artificial cell to the bulk solution. At the steady state, the system can achieve a $\mathrm{pH}$ difference of 0.52 . The effect is due to coupling between the plasmon resonance of the $\mathrm{Au}-\mathrm{Ag}$ nanorods and that of rhodopsin. A second artificial cell containing an ATP synthase within a lipidic membrane uses the difference in proton concentration now existing between the outside and inside compartments to drive the synthesis of ATP molecules.

https://doi.org/10.1038/s41565-019-0408-Z form of adenosine triphosphate (ATP). The
TOPOLOGICAL MATTER

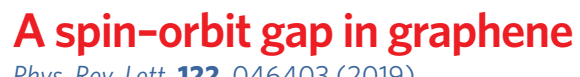

Phys. Rev. Lett. 122, 046403 (2019)

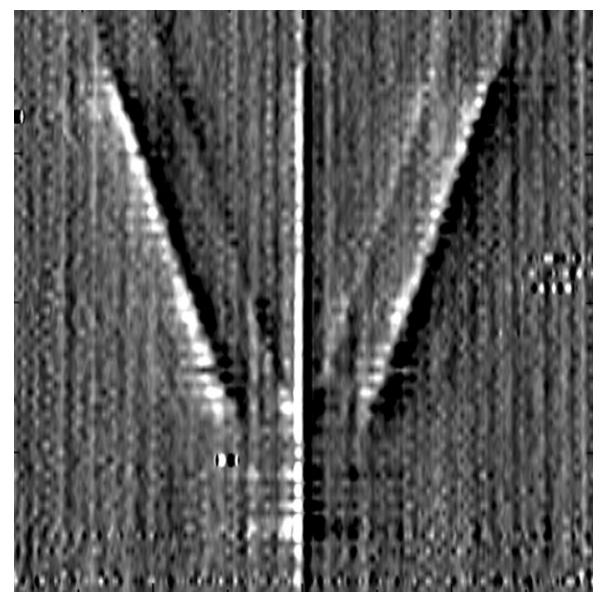

Credit: APS

Graphene is commonly considered a zerogap semiconductor with a Dirac-like band dispersion at the $\mathrm{K}$ and $\mathrm{K}^{\prime}$ points of the Brillouin zone. Yet, at low temperature, the spin-orbit interaction should open a tiny bandgap in the bulk. Only the sample edges would then hold spin-polarized bands connecting the electron bands at $\mathrm{K}$ with the hole bands at $\mathrm{K}^{\prime}$ and vice versa. Sichau et al. now provide experimental evidence for this predicted topologically non-trivial state of graphene.

The researchers produce a graphene device that minimizes sources of extrinsic spin-orbit coupling. Then they measure
ENERGY HARVESTING

\section{Do-it-yourself thermoelectrics}

Converting waste heat into electricity through wearable thermoelectric solid-state devices is an attractive and inexpensive approach to green power generation. Although the performance of organic thermoelectric devices lags far behind that of inorganic counterparts, the abundance and non-toxicity of carbon-based materials that can be solution-processed at low temperatures simplify the fabrication process and reduce the cost of organic thermoelectrics. Now, Abol-Fotouh et al. demonstrate the growth of cellulose films from bacteria embedded with finely dispersed carbon nanotubes to produce a biodegradable thermoelectric paper.

The researchers grow the films in an aqueous solution, which simultaneously serves to stimulate the bacterial growth and disperse colloidally stable nanotubes. After a few days, flexible centimetre-size free-standing films are fully formed. As expected, the films have a low thermal conductivity $\left(1 \mathrm{~W} \mathrm{~m}^{-1} \mathrm{~K}^{-1}\right)$ and, despite a modest carbon nanotube content of just 10 weight per cent, their thermoelectric performance is comparable to that of buckypaper. The composite developed by Abol-Fotouh et al. is p-type, but it can be easily converted into n-type by doping it with, for example, polyethyleneimine. Furthermore, the cellulose can be decomposed using heat or enzymes to collect and recycle the nanotubes. the sample resistance as a function of applied microwave excitation frequency in a magnetic field. In this electron spin resonance (ESR) experiment, the resonant microwave excitation of transitions that couple carriers of opposite spin changes the sample resistance. At zero magnetic field, no transition is allowed, but at higher field, Sichau et al. detect two ESR lines. The frequency difference between these lines corresponds to the energy gap (42.2 $\mu \mathrm{eV}$ ) induced by the spin-orbit coupling, in agreement with some of the earlier theoretical predictions.

https://doi.org/10.1038/s41565-019-0409-y

\section{CANCER NANOMEDICINE}

\section{Liver immunomodulation}

Nat. Commun. 10, 574 (2019)

The liver is characterized by a highly immunosuppressive environment, which hampers the successful application of immunotherapies against tumour and metastasis development.Since a major role in liver immunoregulation is played by liver sinusoidal epithelial cells (LSEC), modulating their activity through targeted intervention might improve the success of immunotherapies. Towards this end, it has been shown that $\alpha$-melittin, a cationic peptide endowed with immunomodulatory properties and tumour cell cytotoxicity, has a signature sequence that recognizes LSEC. However, delivered as free drug, it causes haemolysis.

Now, Yu et al. show that a self-assembled a-melittin-based lipid nanoparticle can be injected intravenously with reduced side effects and delivered specifically to LSEC, where it triggers molecular processes that eventually modulate the liver's immunological state.

Using different in vivo models, the researchers also show that treatment with the nanoparticle prolongs the animals' survival, induces anti-tumour immune memory and reduces the number of metastases without compromising liver functions. In particular, in a spontaneous liver metastastic model, $80 \%$ of the animals survive and are metastasis-free 100 days after primary tumour resection, in contrast to $20 \%$ observed in controls. 